KYUNGPOOK Math. J. 56(2016), 1259-1265

http://dx.doi.org/10.5666/KMJ.2016.56.4.1259

pISSN 1225-6951 eISSN 0454-8124

(c) Kyungpook Mathematical Journal

\title{
On Mean Open and Closed Sets
}

AJoy MukHarJeE*

Department of Mathematics, St. Joseph's College, Darjeeling, W. Bengal- 734 104, India

e-mail : ajoyjee@gmail.com

KALLOL BHANDHU BAGCHI

BSNL Telephone Office Main Road, B.Ed. College Para, Kadamtala, Siliguri, W. Bengal- 734 011, India

e-mail : kbagchi.789@gmail.com

ABstract. In this paper, we introduce the notions of mean open and closed sets in topological spaces, and obtain some properties of such sets. We observe that proper paraopen and paraclosed sets are identical to mean open and closed sets respectively.

\section{Introduction}

Unless otherwise mentioned, $X$ stands for the topological space $(X, \mathscr{P})$. By a proper open set (resp., closed set) of a topological space $X$, we mean an open set $G \neq \emptyset, X$ (resp., a closed set $E \neq \emptyset, X$ ).

During the past few years, maximal and minimal open and closed sets due to Nakaoka and Oda $[3,4,5]$ have been widely investigated in the literature. A nonempty open set $U$ of $X$ is said to be a minimal open set [5] if $U$ and $\emptyset$ are only open sets contained in $U$. Dualizing the notion of minimal open sets, Nakaoka and Oda [4] introduced the notion of maximal open sets: a proper open set $U$ of $X$ is said to be a maximal open set [4] if $X$ and $U$ are only open sets containing $U$. It is seen that if $G$ is a minimal open set and $H$ is a maximal open set in $X$, then either $G \subset H$ or $X$ is disconnected [2, Theorem 2.6]. If $G \subset H$ for a minimal open set $G$ and maximal open set $H$ of a topological space $X$, then there is a possibility to exist an open set $U$ such that $G \subset U \subset H$. Gazing at maximal and minimal open sets, Ittanagi and Benchalli [1] introduced the notion of paraopen sets: an open set of a topological space $X$ is said to be paraopen if it is neither maximal open nor

* Corresponding Author.

Received June 10, 2016; accepted October 21, 2016.

2010 Mathematics Subject Classification: 54A05, 54C05.

Key words and phrases: Mean open set, mean closed set, minimal open set, maximal open set, maximal closed set. 
minimal open. We note that if $V$ is a paraopen set, then there exist proper open sets $G, H(\neq V)$ such that $G \subset V \subset H$ (Theorem 2.5). We observe that $U$ and $V$ as describe above lie between two open sets in the inclusion sense like the arithmetic mean of two real numbers which instigates us to introduce the notion of mean open sets (Definition 3.1).

Throughout the paper, $R$ stands for the set of real numbers.

\section{Some Rectifications}

Firstly, we look at the following two results due to Ittanagi and Benchalli [1].

Theorem 2.1(Theorem 2.5 [1]). Let $X$ be a topological space and $U$ be a nonempty paraopen subset of $X$. Then there exists a minimal open set $N$ such that $N \subset U$.

Theorem 2.2(Theorem 2.6 [1]). Let $X$ be a topological space and $U$ be a proper paraopen subset of $X$. Then there exists a maximal open set $M$ such that $U \subset M$.

In view of Theorem 2.3 (resp., Theorem 2.4), Theorem 2.1 (resp.,, Theorem 2.2 ) holds good if $U$ is a nonempty finite open set (resp., $U$ is a proper nonempty cofinite open set). The complement of a finite subset is termed as a cofinite subset.

Theorem 2.3(Theorem 3.1 [5]). Let $V$ be a nonempty finite open set. Then there exists at least one (finite) minimal open set $U$ such that $U \subset V$.

Theorem 2.4(Theorem 2.7 [4]). Let $V$ be a proper nonempty cofinite open set of $X$. Then there exists at least one (cofinite) maximal open set $U$ such that $V \subset U$.

We consider the usual topology $\mathscr{S}$ on the set $R$. In the topological space $(R, \mathscr{S}),(-1,1)$ is a paraopen set and it is neither contained in a maximal open set nor contains a minimal open set.

In tune of Theorems 2.1 and 2.2, we give the following modified result.

Theorem 2.5. If $V$ is a proper paraopen set, then there exist two proper open sets $G, H(\neq V)$ such that $G \subset V \subset H$.

Proof. Easy to follow from the definition of paraopen sets.

We recall now the following definitions.

Definition 2.6(Nakaoka and Oda [3]). A proper nonempty closed set $E$ of $X$ is said to be a maximal closed set if $X$ and $E$ are only closed sets containing $E$.

Definition 2.7(Nakaoka and Oda [3]). A proper nonempty closed set $E$ of $X$ is said to be a minimal closed set if $E$ and $\emptyset$ are only closed sets contained in $E$.

Definition 2.8(Ittanagi and Benchalli [1]). A closed set $F$ of a topological space $X$ is said to be paraclosed if $X-F$ is paraopen. 
We look at the following two results due to Ittanagi and Benchalli [1].

Theorem 2.9(Theorem $2.9[1])$. Let $X$ be a topological space and $E$ be a nonempty paraclosed subset of $X$. Then there exists a minimal closed set $D$ such that $D \subset E$.

Theorem 2.10(Theorem 2.10 [1]). Let $X$ be a topological space and $E$ be a proper paraclosed subset of $X$. Then there exists a maximal closed set $F$ such that $E \subset F$.

Due to duality principles (i) $F$ is a minimal closed set in $X$ iff $X-F$ is a maximal open set in $X$ [3] and (ii) $F$ is a maximal closed set in $X$ iff $X-F$ is a minimal open set in $X$ [3], Theorems 2.9 and 2.10 do not hold. In tune of Theorems 2.9 and 2.10 , we give the following modified result.

Theorem 2.11. If $E$ is a proper paraclosed set, then there exist two proper closed sets $D, F(\neq E)$ such that $D \subset E \subset F$.

\section{Mean Open and Closed Sets}

Definition 3.1. An open set $M$ of a topological space $X$ is said to be a mean open set if there exist two distinct proper open sets $U, V(\neq M)$ such that $U \subset M \subset V$.

Note that all open sets of a topological space $X$ except $\emptyset$ and $X$ should be mean open sets if $U$ and $V$ are not restricted to be proper open sets in the above definition.

Example 3.2 (Steen and Seebach [6]). Let $X$ be an infinite set and $a \in X$. We define

$$
\mathscr{T}=\{\emptyset\} \bigcup\{G \mid a \in G \subset X\} .
$$

In the topological space $(X, \mathscr{T}),\{a\}$ is not a mean open set and all finite open sets contain at least two elements of $X$ are mean open sets of the space.

The union and intersection of mean open sets may not be mean open sets. In the topological space of Example 3.2, $\{a, b\}$ and $\{a, c\}$ where $b, c \in X$ and $b \neq c \neq a$ are mean open sets but their intersection $\{a\}$ is not a mean open set. For different $b, c, d \in X$ with $b, c, d \neq a, X-\{b, c\}$ and $X-\{c, d\}$ are mean open sets but their union $X-\{c\}$ is not a mean open set.

Definition 3.3. A closed set $E$ of a topological space $X$ is said to be a mean closed set if there exists two distinct proper closed sets $D, F(\neq E)$ such that $D \subset E \subset F$.

Theorem 3.4. An open set of a topological space is a mean open set iff its complement is a mean closed set.

Proof. Let $M$ be a mean open set in $X$. Then we have open sets $U \neq \emptyset, M$ and $V \neq M, X$ such that $U \subset M \subset V$ and so $X-V \subset X-M \subset X-U$. Since $X-V \neq \emptyset, X-M$ and $X-U \neq X-M, X, X-M$ is a mean closed set. 
Conversely, let $M$ be an open set such that $X-M$ is a mean closed set. Hence there exist closed sets $D \neq \emptyset, X-M$ and $F \neq X-M, X$ such that $D \subset X-M \subset F$. It means that $X-F \subset M \subset X-D$. Since $X-F \neq \emptyset, M$ and $X-D \neq M, X, M$ is a mean open set.

Theorem 3.5. A proper paraopen set is a mean open set and vice-e-versa.

Proof. If $P$ is a proper paraopen set, then it is easy to follow by Theorem 2.5 that $P$ is a mean open set.

Conversely, let $M$ be a mean open set in $X$. Then there exist proper open sets $G, H \neq M$ such that $G \subset M \subset H$. Since $G \neq \emptyset, M$ and $H \neq X, M, M$ is neither a minimal nor a maximal open set. As $M \neq \emptyset, X, M$ is a proper paraopen set.

Theorem 3.6. A proper paraclosed set is a mean closed set and vice-e-versa.

Proof. If $E$ is a proper paraclosed set, then it is easy to follow by Theorem 2.11 that $E$ is a mean closed set.

Conversely, let $E$ be a mean closed set in $X$. We have proper closed sets $D, F \neq E$ such that $D \subset E \subset F$. Since $D \neq \emptyset, E$ and $F \neq X, E, E$ is neither a minimal nor a maximal closed set. As $E \neq \emptyset, X, E$ is a proper paraclosed set.

Theorem 3.7(Nakaoka and Oda [4]). If $U$ is a maximal open set and $W$ is an open set, then either $U \cup W=X$ or $W \subset U$. If $U$ and $V$ are two distinct maximal open sets, then $U \cup V=X$.

Theorem 3.8(Nakaoka and Oda [5]). If $U$ is a minimal open set and $W$ is an open set, then either $U \cap W=\emptyset$ or $U \subset W$. If $U$ and $V$ are two distinct minimal open sets, then $U \cap V=\emptyset$.

Theorem 3.9(Mukharjee [2]). If $H$ is a maximal open set and $G$ is a minimal open set of a topological space $X$, then either $G \subset H$ or the space is disconnected.

Theorem 3.10. Let a connected topological space $X$ contain a maximal open set $H$, a minimal open set $G$ with $G \neq H$ and a proper open set $U \neq G, H$. Then only one of the following is true on $X$ :

(i) $U$ is a mean open set such that $G \subset U \subset H$.

(ii) $G \subset X-U \subset H$.

(iii) $G \subset U, H \cup U=X$ and $H \cap U \neq \emptyset$.

(iv) $U \subset H, G \cap U=\emptyset$ and $G \cup U \neq X$.

Proof. Due to Theorem 3.9, we have $G \subset H$. Since $G$ is a minimal open set and $H$ is a maximal open set, we have $G \subset U$ or $G \cap U=\emptyset$ and $U \subset H$ or $H \cup U=X$. The feasible combinations are (i) $G \subset U \subset H$, (ii) $G \cap U=\emptyset$ and $H \cup U=X$, (iii) $G \subset U$ and $H \cup U=X$, (iv) $G \cap U=\emptyset$ and $U \subset H . G \cap U=\emptyset$ and $H \cup U=X$ imply that $G \subset X-U \subset H$. If $G \subset U$ and $H \cup U=X$, then $\emptyset \neq G \subset H \cap U$ since $G \subset H$. If $G \cap U=\emptyset$ and $U \subset H$, then $G \cup U \subset H \neq X$ since $G \subset H$. 
If both (i) and (ii) are true, then we see that $G \subset U \cup(X-U) \subset H$ and $G \subset U \cap(X-U) \subset H . G \subset U \cup(X-U) \subset H$ gives $G \subset X \subset H$ and then we get $H=X$, an absurd result. Also $G \subset U \cap(X-U) \subset H$ gives $G \subset \emptyset \subset H$ and then we get $G=\emptyset$, again an absurd result.

If both (i) and (iii) are true, then $U \subset H$ and $H \cup U=X$ give $H=X$, an absurd result. result.

If both (i) and (iv) are true, then $G \subset U$ and $G \cap U=\emptyset$ give $G=\emptyset$, an absurd

If both (ii) and (iii) are true, then $G \subset X-U$ and $G \subset U$ give $G \subset(X-U) \cap U=$ $\emptyset$ and thus $G=\emptyset$, an absurd result.

If both (ii) and (iv) are true, then $X-U \subset H$ and $U \subset H$ give $(X-U) \cup U=$ $X \subset H$ and thus $H=X$, an absurd result.

If both (iii) and (iv) are true, then we get $G \subset U \subset H, H \cup U=X$ and $G \cap U=\emptyset . U \subset H$ and $H \cup U=X$ give $H=X$, an absurd result. $G \subset U$ and $G \cap U=\emptyset$ give $G=\emptyset$, again an absurd result.

Theorem 3.11. Let a connected topological space $X$ contain a maximal closed set $F$, a minimal closed set $D$ with $D \neq F$ and a proper closed set $E \neq D, F$. Then only one of the following is true on $X$ :

(i) $E$ is a mean closed set such that $D \subset E \subset F$.

(ii) $D \subset X-E \subset F$.

(iii) $E \subset F, D \cap E=\emptyset$ and $D \cup E \neq X$.

(iv) $D \subset E, F \cup E=X$ and $F \cap E \neq \emptyset$.

Proof. We see that the connected topological space $X$ contains a maximal open set $X-D$, a minimal open set $X-F$ and a proper open set $X-E$ with $X-F \neq X-D$ and $X-E \neq X-D, X-F$. By Theorem 3.10, only one of the following is true:

(i) $X-E$ is a mean open set such that $X-F \subset X-E \subset X-D$ which in turn implies that $D \subset E \subset F$. By Theorem 3.4, we see that $E$ is a mean closed set.

(ii) $X-F \subset X-(X-E) \subset X-D$ i.e., $D \subset X-E \subset F$.

(iii) $X-F \subset X-E,(X-D) \cup(X-E)=X$ and $(X-D) \cap(X-E) \neq \emptyset$ i.e., $E \subset F, D \cap E=\emptyset$ and $D \cup E \neq X$.

(iv) $X-E \subset X-D,(X-F) \cap(X-E)=\emptyset$ and $(X-F) \cup(X-E) \neq X$ i.e., $D \subset E$, $F \cup E=X$ and $F \cap E \neq \emptyset$.

Theorem 3.12. If there are two distinct maximal open sets and a mean open set in a topological space, then the intersection of the two maximal open sets is nonempty.

Proof. Let $U, V$ be two distinct maximal open sets and $G$ be a mean open set in a topological space $X$. By Theorem 3.7, $U \cup V=X$. G being a mean open set, 
it is neither maximal open nor minimal open which means that $G \neq U, V$. Also $G \neq \emptyset, X$. By Theorem 3.7, we get $G \varsubsetneqq U$ or $G \cup U=X$ and $G \varsubsetneqq V$ or $G \cup V=X$. The feasible possibilities are (I) $G \varsubsetneqq U$ and $G \varsubsetneqq V$, (II) $G \varsubsetneqq U$ and $G \cup V=X$, (III) $G \cup U=X$ and $G \varsubsetneqq V$ and (IV) $G \cup U=X$ and $G \cup V=X$.

Case I: Obviously, $U \cap V \neq \emptyset$ if $G \varsubsetneqq U$ and $G \varsubsetneqq V$.

Case II: If $G \cap V \neq \emptyset$, then obviously $U \cap V \neq \emptyset$. Now suppose $G \cap V=\emptyset$. As $G \varsubsetneqq U$, there exists an $x \in U$ such that $x \notin G$. Since $G \cup V=X, x \in V$. So $U \cap V \neq \emptyset$.

Case III: Similar to Case II.

Case IV: $G \cup U=X$ and $G \cup V=X$ imply that $G \cup(U \cap V)=X$ which in turn imply that $G=X$ if $U \cap V=\emptyset$. As $G \neq X$, we have $U \cap V \neq \emptyset$.

Theorem 3.13. If there are two distinct minimal open sets and a mean open set in a topological space $X$, then the union of the two minimal open sets is not equal to $X$.

Proof. Let $U, V$ be two distinct minimal open sets and $G$ be a mean open set in a topological space $X$. By Theorem 3.8, $U \cap V=\emptyset$. $G$ being a mean open set, it is neither maximal open nor minimal open which means that $G \neq U, V$. Also $G \neq \emptyset, X$. By Theorem 3.8, we get $U \varsubsetneqq G$ or $G \cap U=\emptyset$ and $V \varsubsetneqq G$ or $G \cap V=\emptyset$. The feasible possibilities are (I) $U \varsubsetneqq G$ and $V \varsubsetneqq G$, (II) $U \varsubsetneqq G$ and $G \cap V=\emptyset$, (III) $G \cap U=\emptyset$ and $V \varsubsetneqq G$ and (IV) $G \cap U=\emptyset$ and $G \cap V=\emptyset$.

Case I: Obviously, $U \cup V \neq X$ if $U \varsubsetneqq G$ and $V \varsubsetneqq G$ as $G \neq X$.

Case II: If $G \cup V \neq X$, then obviously $U \cup V \neq X$. Now suppose $G \cup V=X$. Since $U \varsubsetneqq G$, there exists an $x \in G$ such that $x \notin U$. As $G \cap V=\emptyset, x \notin V$. $x \notin U, V$ imply that $U \cup V \neq X$.

Case III: Similar to Case II.

Case IV: $G \cap U=\emptyset$ and $G \cap V=\emptyset$ imply that $G \cap(U \cup V)=\emptyset$ which in turn imply that $G=\emptyset$ if $U \cup V=X$. As $G \neq \emptyset$, we have $U \cup V \neq X$.

Dualizing Theorems 3.12 and 3.13, we have Theorems 3.14 and 3.15 respectively. We omit the proofs of these theorems as they are similar to the proofs of corresponding results already establish or can be established in the line of proof of Theorem 3.11.

Theorem 3.14. If there are two distinct maximal closed sets and a mean closed set in a topological space, then the intersection of the two maximal closed sets is nonempty.

Theorem 3.15. If there are two distinct minimal closed sets and a mean closed set in a topological space $X$, then the union of the two minimal closed sets is not equal to $X$.

Acknowledgements. The authors are grateful to the referee for examining the paper carefully and for suggesting some necessary corrections in the paper. 


\section{References}

[1] B. M. Ittanagi and S. S. Benchalli, On paraopen sets and maps in topological spaces, Kyungpook Math. J., 56(1)(2016), 301-310.

[2] A. Mukharjee, On maximal, minimal open and closed sets, Commun. Korean Math. Soc., 30(3)(2015), 277-282.

[3] F. Nakaoka and N. Oda, Minimal closed sets and maximal closed sets, Int. J. Math. Math. Sci., 2006, Article ID 18647, 8 pages, doi: 10.1155/IJMMS/2006/18647.

[4] F. Nakaoka and N. Oda, Some properties of maximal open sets, Int. J. Math. Math. Sci., 2003(21)(2003), 1331-1340.

[5] F. Nakaoka and N. Oda, Some applications of minimal open sets, Int. J. Math. Math. Sci., 27(8)(2001), 471-476.

[6] L. A. Steen and J. A. Seebach (Jr), Counterexamples in Topology, Holt, Rinehart, and Winston, New York, 1970 